Originalien

Monatsschr Kinderheilkd $2021 \cdot$ 169:335-345 https://doi.org/10.1007/s00112-021-01125-9 Eingegangen: 2. November 2020

Angenommen: 8. Januar 2021

Online publiziert: 10. Februar 2021

(c) Springer Medizin Verlag GmbH, ein Teil von Springer Nature 2021

\section{Redaktion}

Berthold Koletzko, München

Thomas Lücke, Bochum

Ertan Mayatepek, Düsseldorf

Norbert Wagner, Aachen

Stefan Wirth, Wuppertal

Fred Zepp, Mainz

Das Saarland war in der ersten Welle der Corona Virus Disease 2019(COVID19)-Pandemie eines der am stärksten betroffenen Bundesländer. Niedergelassene Kinder- und Jugendärzte sind erste Ansprechpartner rund um akute Infektionen, haben aber auch die wichtige Aufgabe, präventive medizinische Maßnahmen (v.a. Vorsorgeuntersuchungen und Schutzimpfungen) während einer Pandemie weiter fortzuführen.

Dieser Beitrag analysiert die Auswirkungen des COVID-19-bedingten Lockdowns (Kalenderwoche [KW] 12-18) auf die Konsultationszahlen von 34 der 53 Praxen (64\%) niedergelassener Kinder- und Jugendmediziner im Saarland. Der Beobachtungszeitraum umfasste die KW 2-26 jeweils für 2019 und 2020.

Der erste Fall einer severe acute respiratory syndrome coronavirus 2(SARSCoV-2)-Infektion im Saarland wurde am 03.03.2020 diagnostiziert. Es folgte ein rascher, anhaltender Anstieg der Infektionsfälle: $\mathrm{Ab}$ dem 11.03.2020 lag die kumulierte Inzidenz der Neuerkrankungen/100.000 Einwohner über dem Bundesdurchschnitt, der erst am 25.09.2020 wieder unterschritten wurde. Ihren vorläufigen Höhepunkt erreichte die 7-Tage-Neuerkrankungsinzidenz am 05.04.2020 mit 82,4/100.000 Einwohner, die Zahl der Hospitalisierungen am 15.04.2020 mit insgesamt 222 Patien-

\author{
Karsten Thei ${ }^{1} \cdot$ Arne Simon $^{2} \cdot$ Norbert Graf $^{2} \cdot$ Tilman Rohrer $^{3}$ \\ ${ }^{1}$ Kinder- und Jugendarztpraxis Theiß, St. Ingbert, Deutschland \\ ${ }^{2}$ Klinik für Pädiatrische Onkologie und Hämatologie, Universitätsklinikum des Saarlandes, Homburg/Saar, \\ Deutschland \\ ${ }^{3}$ Klinik für Allgemeine Pädiatrie und Neonatologie, Universitätsklinikum des Saarlandes, Homburg/Saar, \\ Deutschland
}

\title{
Auswirkungen des Lockdowns während der ersten COVID-19- Welle auf 34 kinder- und jugendärztliche Praxen im Saarland
}

ten, davon 58 in intensivmedizinischer Betreuung.

Am 14.03.2020 (KW 11) wurden Kindertageseinrichtungen sowie Schulen geschlossen, ab dem 18.03.2020 (KW 12) galten umfangreiche Ausgangsbeschränkungen sowie Schließungen von vielen Geschäften und öffentlichen Einrichtungen. Erst ab dem 04.05.2020 (KW 19) folgten erste Lockerungen der Kontaktbeschränkungen mit Wiederaufnahme des Schulbetriebs für Abschlussklassen. Dementsprechend galt in den Kalenderwochen 12-18 ein strenger Lockdown unter Schließung von Bildungseinrichtungen und vielen anderen Einrichtungen.

\section{Methodik}

\section{Fallzahlen aller Praxen}

(hausärztliche Versorgung)

Am 30.06.2020 waren im Saarland 52 Praxen zur vertragsärztlichen Versorgung in der Kinder- und Jugendmedizin zugelassen. Hinzu kommt ein medizinisches Versorgungszentrum (MVZ), das ebenfalls einen hausärztlichen Versorgungsauftrag in der Kinder- und Jugendmedizin erfüllt. Daher ist das MVZ in diese Arbeit wie eine Praxis eingeflossen, was eine Gesamtzahl von 53 Einrichtungen resp. Praxen bedeutet. Über das Intra- net des Berufsverbandes der Kinder- und Jugendärzte (PädInform ${ }^{\mathrm{rx}}$ ) sowie per Fax wurden alle 53 Praxen zur Teilnahme eingeladen und im Verlauf auch nochmals an die Erhebung erinnert.

Mittels der Statistikfunktion des praxiseigenen Arztinformationssystems (AIS) wurden die Gesamtfallzahlen/KW ermittelt. Der Erhebungszeitraum umfasste jeweils die KW 2-26/2020; als Vergleichszeitraum dienten die KW 2-26/ 2019. Unter einem Fall ist/sind hierbei eine oder mehrere Konsultationen im abgefragten Zeitraum zu verstehen, bei der/denen mindestens eine Gebührenordnungsposition (GOP) aus dem Einheitlichen Bewertungsmaßstab (EBM) angesetzt wurde. Mehrere Konsultationen innerhalb einer Kalenderwoche wurden als ein Fall gezählt.

Die Vorgabe war, die Abfrage auf die jeweilige Kalenderwoche und auf die Betriebsstättennummer der Hauptpraxis $\mathrm{zu}$ begrenzen, um Fälle aus Diensten in der Bereitschaftsdienstpraxis (kinderärztlicher Notdienst) auszuschließen. Da diese Funktion nur für Fälle der vertragsärztlichen Versorgung in allen AIS implementiert ist, bleiben Behandlungen aus anderen Versicherungsverhältnissen bzw. Abrechnungsmodalitäten unberücksichtigt.

Zusätzlich abgefragt wurde die Fallzahl von Früherkennungsleistungen 


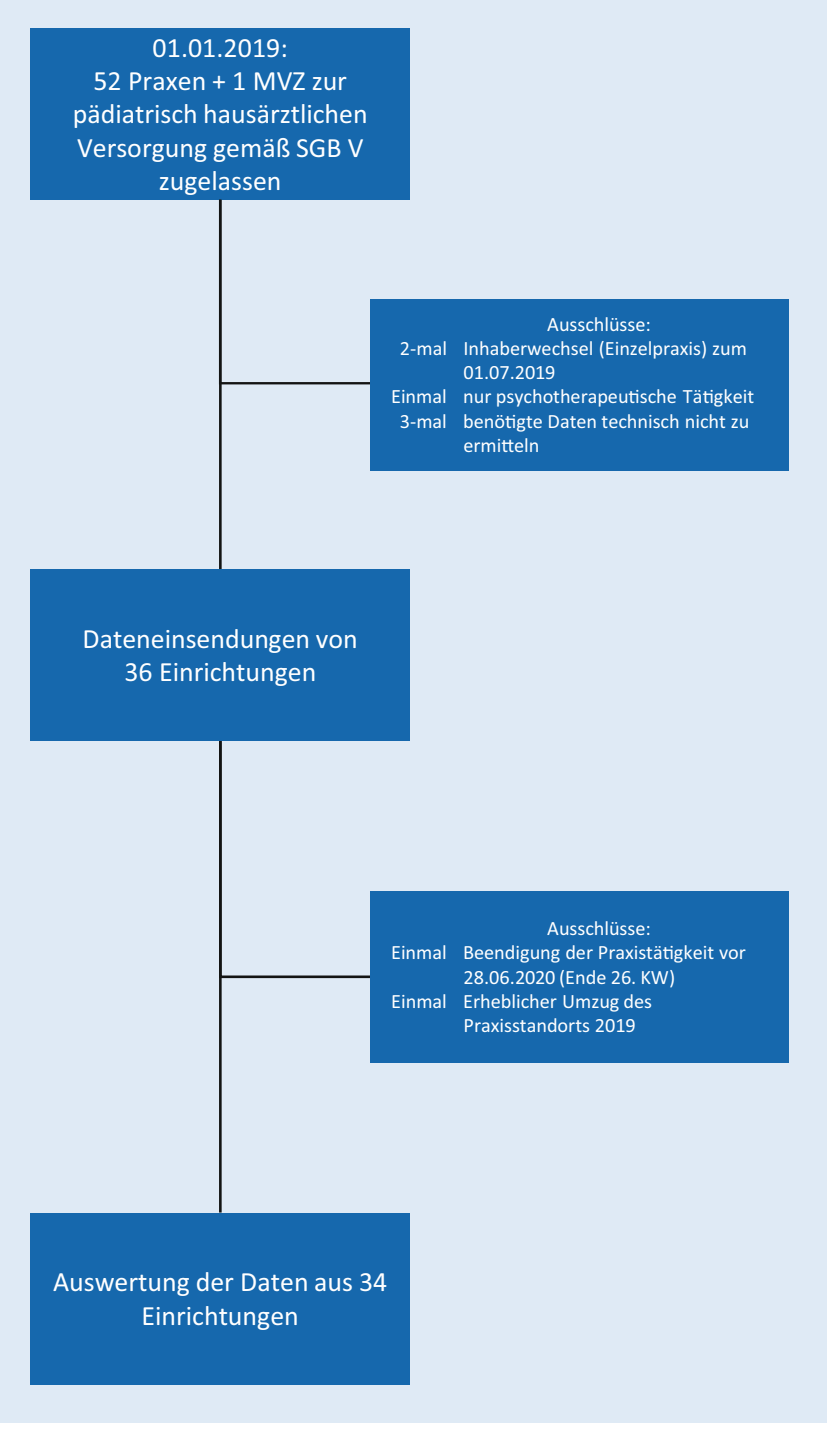

Abb. $1<$ In die Fallzahlauswertung eingeschlossene Praxen
(Konsultationen für Vorsorgeuntersuchungen und, abhängig vom Arztinformationssystem, auch für Impfungen). Unter einem Fall der Früherkennung/ Prävention ist/sind hierbei eine oder mehrere Konsultationen im abgefragten Zeitraum zu verstehen, bei der/denen mindestens eine EBM-GOP aus dem Bereich der Vorsorgeuntersuchungen (GOP 01712-01723) und/oder aus dem Bereich der Impfungen (GOP 89100-89600) abgerechnet wurde. Sind mehrere Leistungen im abgefragten Zeitraum (z.B. Kindervorsorgeuntersuchung U6 mit Impfung gegen Masern-Mumps-Röteln) erbracht worden, so wird dieses als ein Fall gezählt.

In der technischen Umsetzung der Abfrageoptionen gab es Unterschiede in der jeweiligen Praxissoftware, sodass bei einem Teil der Praxen Vorsorgeuntersuchungen und Impfungen dem gleichen Konsultationstyp zugeordnet wurden. Innerhalb einer Praxis sind die Daten aber immer auf die gleiche Weise erhoben worden und konnten daher mit den Ergebnissen von 2019 verglichen werden. In die Auswertung aufgenommen wurden alle Vorsorgeuntersuchungen, die im Kollektivvertragssystem über die kassenärztliche Vereinigung abgerechnet werden. Im konkreten Fall des Saarlandes sind hier nicht die Kindervorsorgeuntersuchungen von Versicherten der Krankenkasse „Barmer“ enthalten, die am Selektivvertrag zur hausarztzentrierten Versorgung gemäß $₫ 73 b$ Sozialgesetzbuch V teilnehmen.
Die auf diese Weise ermittelten Fallzahlen (kumulative Ergebnisse Gesamtfallzahl und Fallzahl Früherkennung, ohne Sozialdaten der Kinder und Jugendlichen) wurden für die Kalenderwoche in tabellarischer Form an den Erstautor übermittelt. Aus den übermittelten Fallzahlen der Praxen wurden die Fallzahländerungen (absolut und prozentual) pro Kalenderwoche und Praxis berechnet. Durch einzelne Schließtage der Praxen (Fortbildung, tageweiser Urlaub (z.B. 2019 2-Tage-Woche, 2020 5-TageWoche) etc.) entstehen zunächst auch deutliche Ausreißer. Um diesen Effekt, der nicht das wirkliche Konsultationsverhalten darstellt, abzumildern, wurden die Fallzahlen pro Praxis jeweils für die Zeitblöcke KW 2-11 sowie KW 12-18 und KW 19-26 in beiden Vergleichszeiträumen zusammengezählt und aus diesen Summen die Fallzahlveränderungen Prä-Lockdown, im Lockdown und Post-Lockdown ermittelt. Über die Zeiträume von mindestens 7 Wochen sollten oben genannte Phänomene weitestgehend ausgeglichen werden, und die Fallzahländerungen spiegeln auch das Konsultationsverhalten und Arbeitsaufkommen in den Praxen wider.

Die Aufbereitung und Auswertung der Daten erfolgten in Excel $365^{\mathrm{Tm}}$ (Version 2009, Build 13231.20390 Klick-undLos; Microsoft, Redmond, WA, USA). Bei der Erstellung von Boxplots in Microsoft Excel ${ }^{\mathrm{Tm}}$ kommt das Add-in von C. Sekulla aus dem Universitätsklinikum Halle (Saale) zum Einsatz ${ }^{1}$. Es wird für die Quartildefinitionen Q1 und Q3 die Einstellung der Definition nach Tukey (SPSS Boxplot, Tukey-Angelpunkte) gewählt.

\section{Fallzahlen in der fachärztlichen Versorgung („SSpezialpädiater $\left.{ }^{\prime \prime}\right)$}

Kolleginnen und Kollegen, die neben dem allgemeinpädiatrischen, hausärztlichen Versorgungsauftrag auch noch einen fachärztlichen Versorgungsauftrag, sog. Schwerpunktpädiater (z.B. Neuropädiatrie, Kinderkardiologie, pädiatrische Pneumologie etc.), innehaben,

\footnotetext{
1 https://www.medizin.uni-halle.de/index
} php?id=1358 (zugegriffen: 26.07.2020). 
Hier steht eine Anzeige.

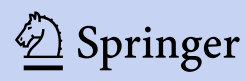


Monatsschr Kinderheilkd 2021 · 169:335-345 https://doi.org/10.1007/s00112-021-01125-9

(c) Springer Medizin Verlag GmbH, ein Teil von Springer Nature 2021

\section{K. Theiß · A. Simon · N. Graf · T. Rohrer}

\section{Auswirkungen des Lockdowns während der ersten COVID-19-Welle auf 34 kinder- und jugendärztliche Praxen im Saarland}

\section{Zusammenfassung}

Hintergrund. Das Saarland war in der ersten Welle der COVID-19-Pandemie eines der am stärksten betroffenen Bundesländer. Niedergelassene Kinder- und Jugendärzte sind erste Ansprechpartner bei Fragen zu Risikofaktoren, Symptomen und Erkrankungsverdacht. Der Lockdown könnte gravierende Auswirkungen auf die allgemeinen Konsultationszahlen, auf Vorsorgeuntersuchungen und Impfungen haben.

Fragestellung. Auswirkungen des COVID19-bedingten Lockdowns im Saarland auf die Patientenversorgung in der ambulanten Pädiatrie bezüglich Gesamtkonsultationen, Früherkennungsuntersuchungen sowie fachärztlicher Versorgung.

Methoden. Alle 53 saarländischen Kinderund Jugendarztpraxen wurden gebeten, ihre
Fallzahlen für die Kalenderwochen 2-26 der Jahre 2019 und 2020 zur Verfügung zu stellen. Diese Daten wurden mittels deskriptiver Statistik analysiert.

Ergebnisse. Die Daten aus 34 (64\%) saarländischen Kinder- und Jugendarztpraxen wurden ausgewertet. Im Lockdown kam es zu einem Rückgang von $-37,0 \%$ in der Gesamtfallzahl, $-9,4 \%$ in der Früherkennungsfallzahl und $-35 \%$ in der fachärztlichen Versorgung (4 Schwerpunktpraxen). Nach dem Lockdown blieb im Vergleich zu 2019 die Gesamtfallzahl mit $-17,1 \%$ deutlich erniedrigt, die Früherkennungsfallzahl holt mit $+9,4 \%$ fast vollständig auf, und auch die fachärztliche Versorgung zeigt mit $+7 \%$ Aufholeffekte.
Diskussion. Die ambulante Pädiatrie konnte im Saarland Lockdown-bedingte Kollateralschäden bezüglich präventiver Leistungen vermeiden und ausgefallene/verschobene Leistungen bereits im Beobachtungszeitraum nachholen. Die Gesamtfallzahlen blieben bis zum Ende des Untersuchungszeitraums niedriger als 2019, hierzu könnte auch der ausgeprägte Rückgang anderer respiratorischer Infektionen durch die SARS-CoV-2Präventionsmaßnahmen beigetragen haben.

Schlüsselwörter

Respiratorische Infektionen · Pädiatrie · Ambulante Versorgung · Vorsorgeuntersuchungen . Gastroenteritis

\section{Consequences of the lockdown during the first COVID-19 wave in 34 pediatric practices in Saarland}

\section{Abstract}

Background. In the first COVID-19 pandemic wave Saarland was one of the most affected federal states. Pediatric practices are the primary point of contact regarding questions about risk factors, symptoms and suspicion of illness. The lockdown could have a severe impact on overall number of consultancies, preventive medical check-ups and vaccinations.

Objective. The impact of COVID-19-related lockdowns in Saarland regarding patient care in the ambulatory pediatric setting and general consultation, early preventive consultancy as well as specialized medical care.

Material and methods. All 53 pediatric practices in Saarland were asked to report their case numbers for calendar weeks 2-26 in 2019 and 2020 . These data were analyzed via descriptive statistics.

Results. Data from 34 (64\%) of Saarland's pediatric practices were evaluated. There was a decline of $-37 \%$ regarding overall cases during lockdown, $-9.4 \%$ of early diagnosis cases and $-35 \%$ regarding specialized medical consultancy (4 specialized practices). After lockdown the overall case numbers were significantly reduced with $-17.1 \%$ in comparison to 2019 , the early diagnosis cases caught up nearly completely with $+9.4 \%$ and also the specialized care showed catch-up effects with $+7 \%$.

Conclusion. Lockdown-related collateral damage regarding preventive performan- ces/activities could be avoided in the ambulatory pediatric setting in Saarland. Cancelled/postponed activities caught up during the observation period.

During the observation period, the overall case numbers remained lower than 2019. The reason could be the contribution of SARSCoV-2 prevention measures to significantly reduce other respiratory infections.

\section{Keywords} Respiratory Tract Infections · Pediatrics · Outpatient treatment $\cdot$ Screening investigations . Gastroenteritis wurden zusätzlich gebeten, die Anzahl der abgerechneten fachärztlichen Versichertenpauschalen (EBM-GOP 04000F bzw. 04001F und 04002F) pro Kalenderwoche anzugeben. Das weitere Vorgehen (Vergleichszeiträume, Datenübermittlung) war identisch $\mathrm{zu}$ den vorherigen Ausführungen.

\section{Ermittlung der Infektions- konsultationen}

Ergänzend erhob die Praxis des Erstautors die Konsultationen aufgrund respiratorischer Infektionen und Gastroenteritiden im Jahresvergleich sowie durchgeführte SARS-CoV-2-realtime polymerase chain reaction(rtPCR)-Abstriche in 2020. Für die respiratorischen Infekte wurde nach Fällen gesucht, bei denen eine gesicherte Diagnose aus dem Inter- national Statistical Classification of Diseases and Related Health Problems (10th Revision) (ICD-10)-Katalog von J00-J22 dokumentiert wurde. Die Patienten, die wegen mehrerer Manifestationen an den Atemwegen in gleicher Sitzung behandelt wurden, wurden entsprechend nur als eine Konsultation gezählt. Wurde der Patient jedoch in der gleichen Woche mehrmals an verschiedenen Tagen behandelt, so wurde jede Konsultation einzeln gezählt. 


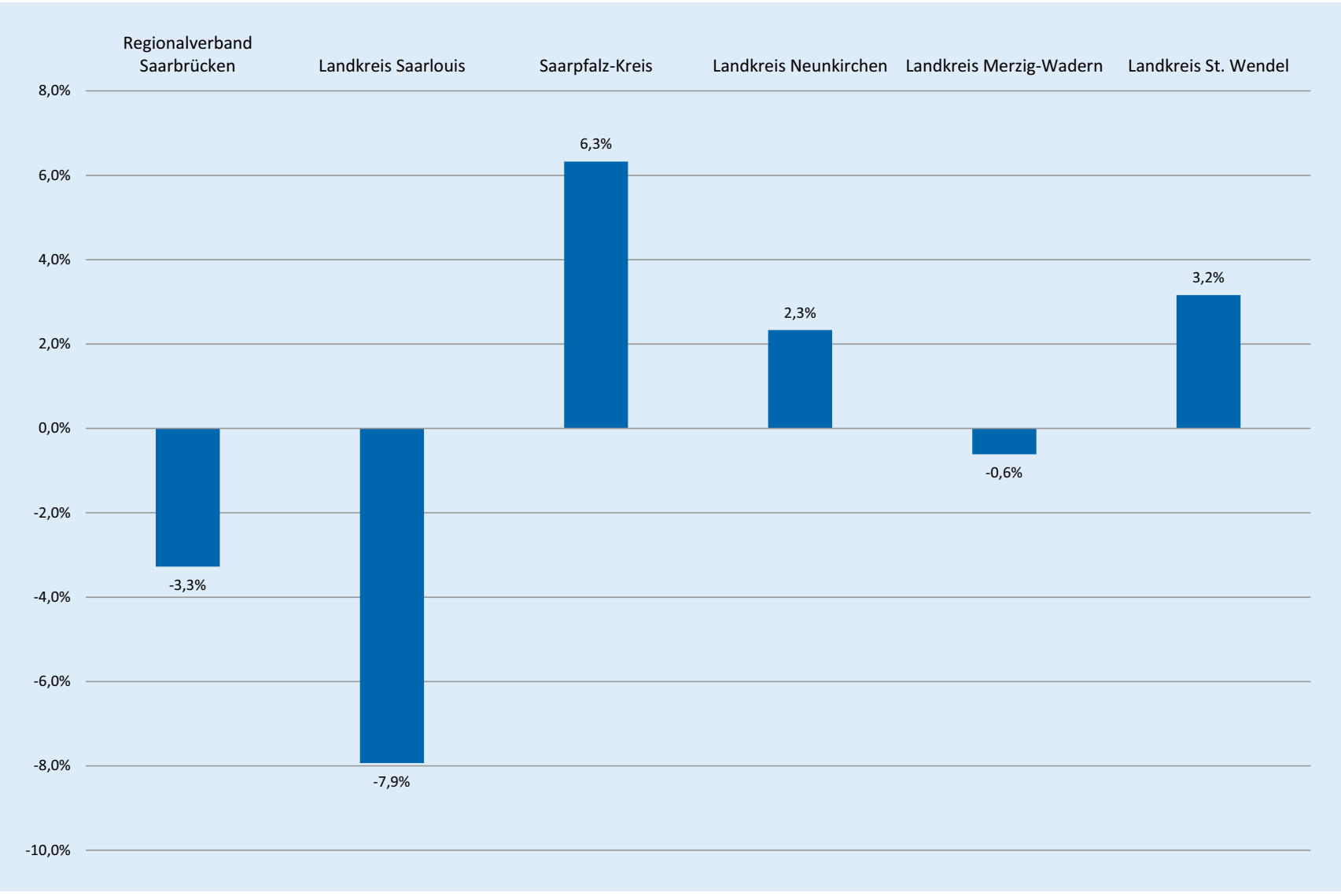

Abb. 2 A Abweichung zwischen Zulassung und ausgewerteten Praxen:Differenz der Prozentpunkte zwischen der Verteilung der Praxen gemäß Zulassung sowie der ausgewerteten Praxen auf die Landkreise bzw. den Regionalverband Saarbrücken

Bezüglich der Gastroenteritiden erfolgte die Identifikation der Fälle über die gesicherte Diagnose „A09“ nach ICD-10. Da in der Praxis keine Erregerdiagnostik bei Gastroenteritiden durchgeführt wird, sind diese sämtlich als A09.0 verschlüsselt.

Für die Konsultationen von COVID19(Verdachts)-Fällen erfolgte ebenfalls die analoge Identifikation aller Konsultationen, bei denen eine SARS-CoV-2PCR-Diagnostik durchgeführt bzw. veranlasst wurde. Da es sich hier i.d.R. um Atemwegsinfektionen handelt, sind diese Patienten zugleich auch in der Kohorte „respiratorische Infekte“ enthalten.

Aus den absoluten Konsultationszahlen wurde ein Konsultationsindex berechnet. Dieser gibt den Anteil an Patienten mit respiratorischen Infektionen, Gastroenteritiden bzw. COVID-19(Verdachts)-Fällen als Anteil an den Gesamtkonsultationen der Woche an. Durch diese Indexbildung können passagere Veränderungen des Sprechstundenumfangs (z. B. Fortbildungstage) und damit Differenzen zwischen den beiden Jahren mitabgebildet werden.

Die Ethikkommission der Ärztekammer des Saarlandes erteilte für diese Untersuchungen ein positives Ethikvotum (Kenn-Nr.: 188/20).

\section{Ergebnisse}

\section{Stichprobe}

Von den 53 eingeladenen Praxen haben sich 42 Praxen zurückgemeldet (79\%). 2 Praxen wurden ausgeschlossen, da es zwischen den beiden Vergleichszeiträumen zu einem Inhaberwechsel in den Einzelpraxen gekommen ist. 1 Praxis ist ausschließlich psychotherapeutisch tätig, und bei 3 Praxen war es nicht möglich, die erforderlichen Daten im Arztinformationssystem zu erhalten.

Ingesamt haben 36 Praxen (68\%) die Fallzahlen gemäß den Vorgaben an den Erstautor übermittelt, wobei 2 Praxen von der weiteren Auswertung ausgeschlossen wurden. Eine Praxis hat die Tätigkeit vor Ende des Beobachtungszeitraums 2020 eingestellt, die andere Praxis ist 2019 an einen neuen Standort mit überwiegendem Wechsel des Patientenkollektivs gezogen und konnte daher keine vergleichbaren Daten beitragen. Letztendlich wurden somit Daten aus 34 Praxen (64\%) in die Auswertung einbezogen (• Abb. 1).

Die ausgewerteten Praxen waren hinsichtlich ihrer verwaltungsgeografischen Lage der Praxisstandorte in den Landkreisen bzw. dem Regionalverband Saarbrücken gut mit der Verteilung der $\mathrm{Zu}$ lassungen vergleichbar (• Abb. 2).

Die teilnehmenden Praxen haben 7 verschiedene Softwareprodukte als Arztinformationssysteme im Einsatz (- Tab. 1). In 25 Praxen (74\%) kommen AIS zum Einsatz, die als Früherkennungsfallzahl ausschließlich Vorsorgeuntersuchungen auswerten, und in 9 Praxen (26\%) AIS, die sowohl Vorsor- 
Tab. 1 Verteilung der eingesetzten Arztinformationssysteme in der Stichprobe

\begin{tabular}{|c|c|c|c|}
\hline Softwareprodukt & Hersteller \& Vertrieb & Definition der Früherkennung & Anzahl der Praxen \\
\hline CGM MEDISTAR ${ }^{\mathrm{TM}}$ & CompuGroup Medical Deutschland AG, Koblenz & Vorsorgen & 17 \\
\hline$x$. concept $^{\mathrm{TM}}$ & Medatixx GmbH \& Co. KG, Eltville/Rhein & Vorsorgen und/oder Impfungen & 8 \\
\hline x.isynet $^{\mathrm{TM}}$ & Medatixx GmbH \& Co. KG, Eltville/Rhein & Vorsorgen & 4 \\
\hline MEDICAL OFFICE ${ }^{\mathrm{TM}}$ & $\begin{array}{l}\text { INDAMED EDV-Entwicklung und -Vertrieb GmbH, Schwe- } \\
\text { rin }\end{array}$ & Vorsorgen und/oder Impfungen & 1 \\
\hline Medatixx ${ }^{\mathrm{TM}}$ & Medatixx GmbH \& Co. KG, Eltville/Rhein & Vorsorgen & 2 \\
\hline$S 3^{\mathrm{TM}}$ & S3 Praxiscomputer GmbH, Weinheim & Vorsorgen & 1 \\
\hline CGM M1 PRO ${ }^{\mathrm{TM}}$ & CompuGroup Medical Deutschland AG, Koblenz & Vorsorgen & 1 \\
\hline
\end{tabular}

Tab. 2 Entwicklung der Gesamt- und Präventionsfallzahlen ${ }^{\text {a }}$ in 34 ausgewerteten Praxen vor, während und nach der Lockdown-Phase im Jahr 2020 relativ zu denjenigen derVergleichsperioden im Jahr 2019

\begin{tabular}{|c|c|c|c|}
\hline & $\begin{array}{l}\text { Prä-Lockdown } \\
\text { (KW 02-11) }\end{array}$ & $\begin{array}{l}\text { Lockdown } \\
\text { (KW 12-18) }\end{array}$ & $\begin{array}{l}\text { Post-Lockdown } \\
\text { (KW 19-26) }\end{array}$ \\
\hline $\begin{array}{l}\text { Gesamtfallzahl } \\
\text { (\%) }\end{array}$ & $\begin{array}{l}-1,8 \\
(-19,2-+17,5) \\
{[-5,6-+6,1]}\end{array}$ & $\begin{array}{l}-37,0 \\
(-58,5--2,7) \\
{[-42,5--29,5]}\end{array}$ & $\begin{array}{l}-17,1 \\
(-38,6-+11,1) \\
{[-23,5--10,0]}\end{array}$ \\
\hline $\begin{array}{l}\text { Präventions- } \\
\text { fallzahl (\%) }\end{array}$ & $\begin{array}{l}1,4 \\
(-21,0-+22,0) \\
{[-8,6-+10,5]}\end{array}$ & $\begin{array}{l}-9,4 \\
(-64,3-+36,1) \\
{[-24,6-+3,2]}\end{array}$ & $\begin{array}{l}+9,4 \\
(-14,5-+49,2) \\
{[-0,1-+19,6]}\end{array}$ \\
\hline
\end{tabular}

Tab. 3 Entwicklung der Gesamt- und Facharztfallzahlen ${ }^{\text {a } i n ~} 4$ ausgewerteten Praxen mit hausund fachärztlichen Versorgungsauftrag vor, während und nach der Lockdown-Phase im Jahr 2020 relativ zu denjenigen der Vergleichsperioden im Jahr 2019

\begin{tabular}{llll} 
& $\begin{array}{l}\text { Prä-Lockdown } \\
\text { (KW 02-11) }\end{array}$ & $\begin{array}{l}\text { Lockdown } \\
\text { (KW 12-18) }\end{array}$ & $\begin{array}{l}\text { Post-Lockdown } \\
\text { (KW 19-26) }\end{array}$ \\
\hline Gesamtfallzahl (\%) & -1 & -38 & -17 \\
& $(-14-+7)$ & $(-42--33)$ & $(-22--11)$ \\
& {$[-6-+3]$} & {$[-41--34]$} & {$[-19--15]$} \\
Facharztfallzahl (\%) & -2 & -35 & +7 \\
& $(-6-+13)$ & $(-52--30)$ & $(-26-+16)$ \\
& {$[-4-+3]$} & {$[-42--31]$} & {$[-2-+10]$}
\end{tabular}

aMedian, Minimum-Maximum-Spanne (rund Klammern) und Spanne der Quartile 1-3 (eckige Klammern) der prozentualen Fallzahländerung

geuntersuchungen wie auch Impfungen als Früherkennungsfallzahl auswerten.

Vier Praxen haben zusätzlich die Anzahl der abgerechneten fachärztlichen Versichertenpauschalen übermittelt.

\section{Gesamtfallzahlen}

Die kumulativen Gesamtfallzahlen aller Praxen zeigen 2019 und 2020 bis einschließlich KW 11 einen vergleichbaren Verlauf. Ab der KW 12 fallen die Gesamtfallzahlen mit Ausnahme der KW 22 und 25 bis zum Ende des Beobachtungszeitraums im Vergleich zum Vorjahr stark ab. Die Auswertung der Konsultationszahlen vor (KW 2-11), während

\section{Früherkennungsfallzahlen}

Die Früherkennungsleistungen zeigen Anfang 2020 (KW 2-11) mit einer medianen Fallzahlveränderung von $+1,4 \%$ (- Tab. 2) im Verlauf keinen wesentlichen Unterschied zum Vorjahr. Im Lockdown (KW 12-18) kommt es mit $-9,4 \%$ zu einem medianen Rückgang der Fallzahlen. Im Gegensatz zu den Gesamtkonsultationen zeigen die präventiven Konsultationen nach dem Lockdown (KW 19-26) einen medianen Aufholeffekt auf $+9,4 \%$ (• Abb. 3). Die im Lockdown ausgefallenen bzw. verschobenen Vorsorgeleistungen wurden bis Ende des ersten Halbjahres fast vollständig aufgeholt: Summe über alle 34 Praxen: -95 Präventionsfälle $(-0,4 \%)$ bis Ende KW 26 im Vergleich zum Vorjahr. Der Anteil der Früherkennungsfälle an der Gesamtfallzahl lag 2019 minimal bei $11,7 \%$ (KW 9) und maximal bei 19,5\% (KW 26). 2020 lag der Anteil ab der Kalenderwoche 13, mit Ausnahme der KW 16, konstant über $20 \%$. Das Minimum lag 2020 bei 9,9\% (KW 1) und das Maximum bei 25,1\% (KW 20).

(KW 12-18) und nach der LockdownPhase (KW 19-26) zeigt deutliche Unterschiede im Verlauf der Gesamtfallzahlen im Jahresvergleich.

Vor dem Lockdown liegt der Median der Fallzahländerung bei -1,8\% (•Tab. 2), während des Lockdowns hingegen bei $-37,0 \%$. Keine der teilnehmenden Einrichtungen erreicht während des Lockdowns die Konsultationszahlen des Vorjahres. Nach dem Lockdown erholen sich die Konsultationszahlen leicht auf im Median -17,1 \% (• Abb. 3).

\section{Fachärztliche Versorgung (,Spezialpädiater")}

Bezüglich der Gesamtfallzahl vor, während und nach dem Lockdown liegen die 4 Praxen, die zugleich eine fachärztliche Schwerpunktversorgung anbieten, im Median identisch zur Gesamtheit aller Praxen (•Tab. 3). Wenn man sich die Entwicklung der fachärztlichen Fallzahlen näher betrachtet, so ist diese vor dem Lockdown bei einem Median der Fallzahländerung von $-2 \%$ sowie im Lockdown mit $-35 \%$ identisch zur Veränderung der Gesamtfallzahl der Praxen. Nach dem 
Hier steht eine Anzeige.

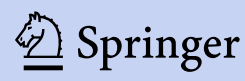




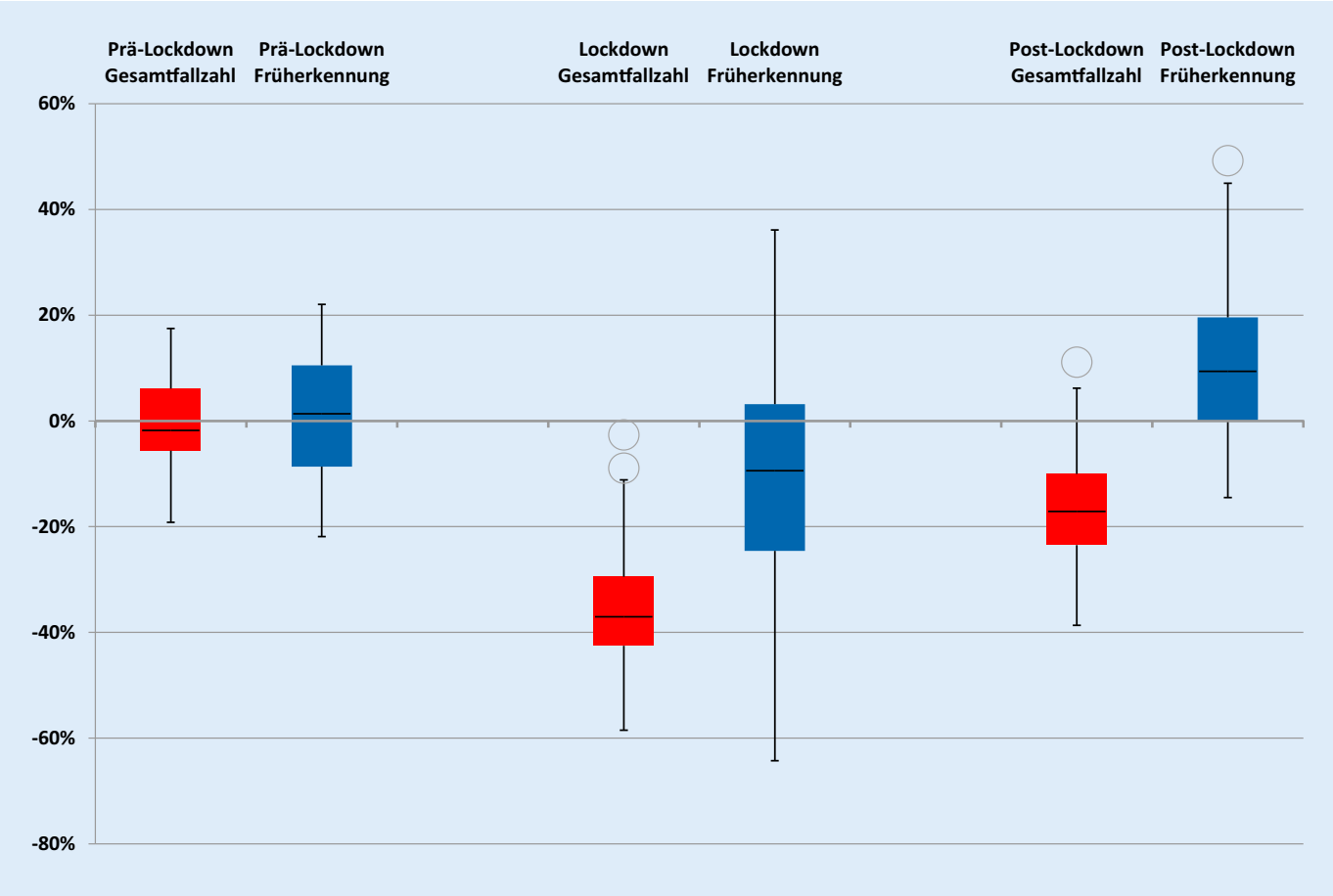

Abb. $3 \triangleleft$ Fallzahlveränderung vor, während und nach dem Lockdown in den ausgewerteten Praxen. rot Gesamtfallzahlen, blau Präventionsfallzahlen

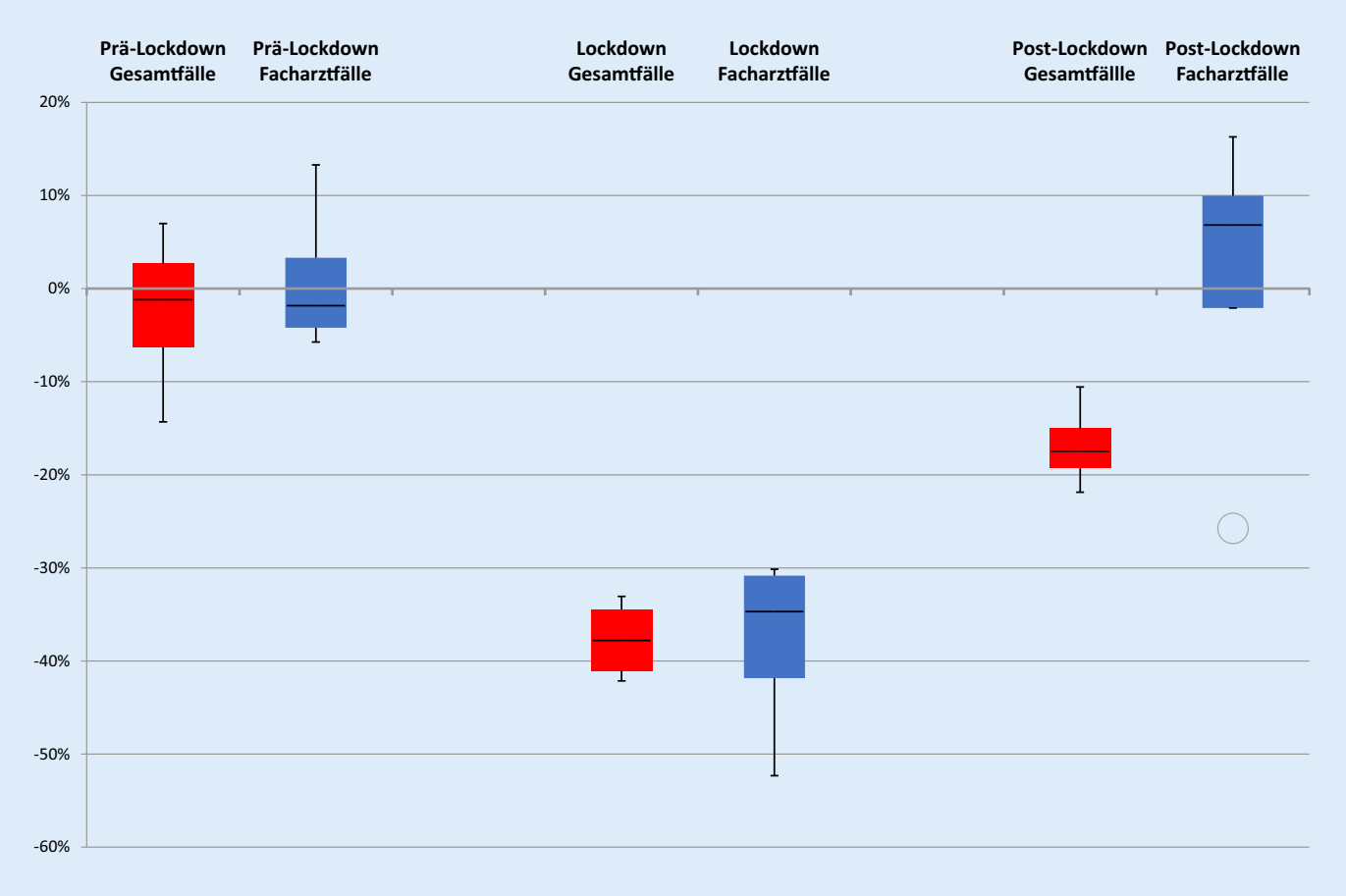

Abb. $4 \triangleleft$ Fallzahlveränderung vor, während und nach dem Lockdown in fachärztlichen Sprechstunden („Spezialpädiater") von 4 teilnehmenden Praxen. rot Gesamtfallzahlen der 4 teilnehmenden Praxen, blau Facharztfallzahlen
Lockdown zeigt sich jedoch im Bereich der fachärztlichen Versorgung ein deutlich rascherer Aufholeffekt. Der Median der fachärztlichen Versorgung liegt nach dem Lockdown bei $+7 \%$, wohingegen in den gleichen Praxen der Median der Gesamtfallzahl noch bei $-17 \%$ liegt (• Tab. 3; - Abb. 4).
Infektionskonsultationen (eine Praxis)

Für die Praxis des Erstautors zeigt sich bis zur KW 12 für Atemwegsinfektionen (• Abb. 5) im Wesentlichen ein identischer Verlauf im Vergleich zu 2019. Ab KW 13 kommt es zu einem starken Abfall und während KW 15-20 im Prinzip zu keinen Vorstellungen wegen respiratorischer Infekte. Ein vergleichbares Bild, ohne Vorstellungen von KW 16-22, ergibt sich bei den Gastroenteritiden.

Nach dem Lockdown haben zunächst die Schulen stufenweise ihren Betrieb wieder aufgenommen, sind bis zu den Sommerferien jedoch nicht zu einem vollständigen Betrieb zurückgekehrt. Ab 


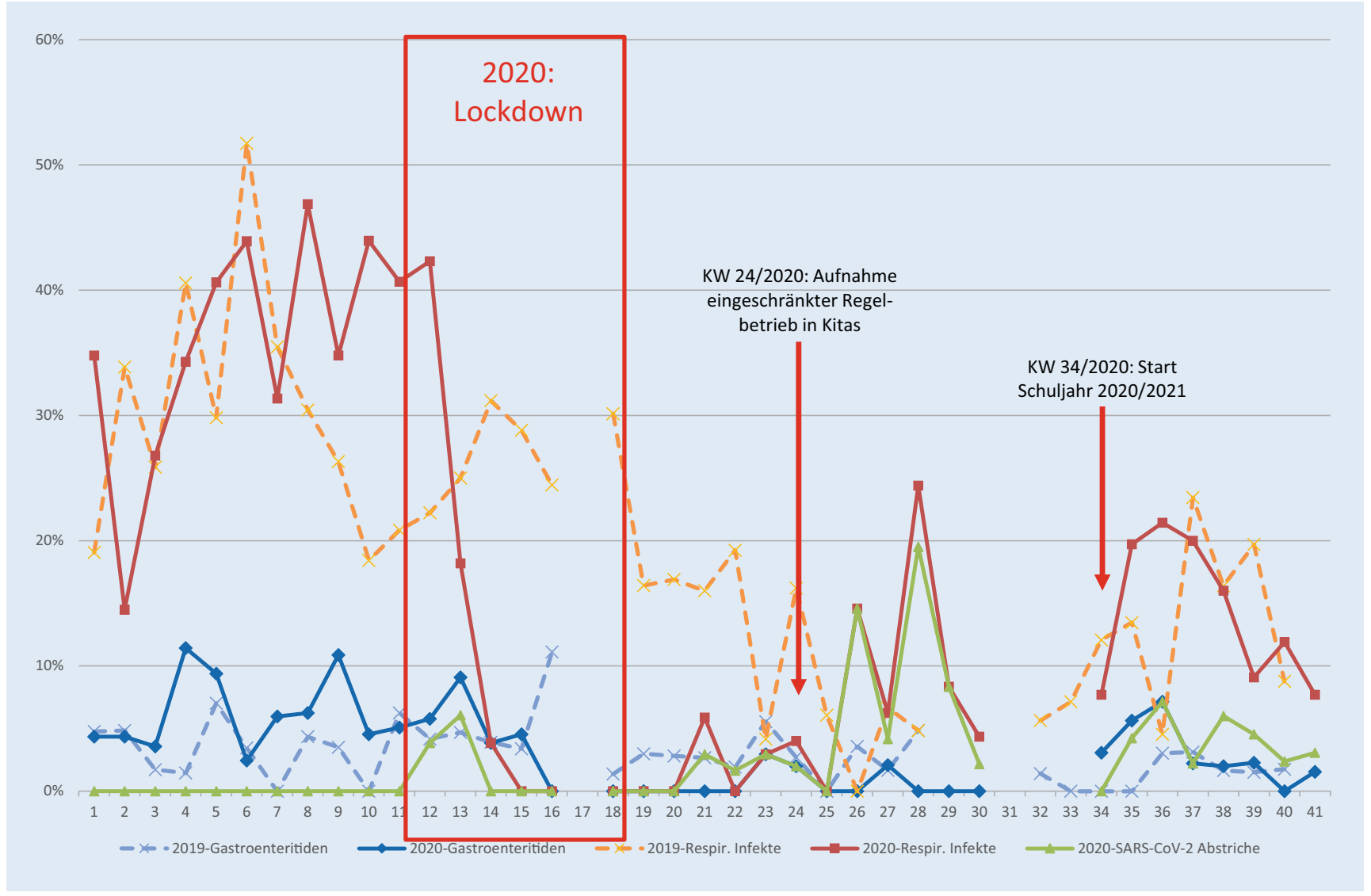

Abb. $5 \Delta$ Respiratorische Infekte (ICD-10-Codes J00-J22) und Gastroenteritiden (ICD-10-Code A09): prozentualer Anteil an der Gesamtfallzahl (Konsultationsindex) in der Praxis des Erstautors vor, während und nach der Lockdown-Phase 2020 und in den Vergleichszeiträumen 2019. Darstellung nach Kalenderwochen und Kennzeichnung der Öffnung von Kindergemeinschaftseinrichtungen im Saarland. Unterbrochene Linie: Urlaub bzw. fehlende Daten

der KW 24 wurde in den Kindertageseinrichtungen (Kita) der eingeschränkte Regelbetrieb wieder aufgenommen, wobei nicht alle Einrichtungen diesen sofort umsetzen konnten und daher teilweise erst eine oder 2 Wochen später gestartet sind. Das Schuljahr 2020/2021 begann in der KW 34 mit einem vollständigen Präsenzschulbetrieb aller Schulen (• Abb. 5).

\section{Testung auf Severe Acute} Respiratory Syndrome Coronavirus 2 (SARS-CoV-2)

Bezüglich derSARS-CoV-2-Abstriche ist anzumerken, dass sich die Indikationen für eine SARS-CoV-2-Testung während der Beobachtungszeit dieser Erhebung mehrfach geändert haben. Zunächst war die Grundlage der Indikationsstellung die RKI-Veröffentlichung „Maßnahmen und Testkriterien - Orientierungshilfe für Ärzte“ [15] in der jeweils gültigen Fassung. Diese Empfehlungen wurden durch das Robert Koch-Institut jeweils am 05.03.2020 (KW 10), 24.03.2020 (KW 13), 06.04.2020 (KW 15), 22.04.2020 (KW 17), 06.05.2020 (KW 19) und zuletzt am 12.05.2020 (KW 20) in aktualisierter Fassung herausgegeben. $\mathrm{Ab}$ der Kalenderwoche 34 war dann die zwischenzeitliche Veröffentlichung unter Federführung der Deutschen Akademie für Kinder- und Jugendmedizin (DAKJ) „Maßnahmen zur Aufrechterhaltung eines Regelbetriebs und zur Prävention von SARS-CoV-2-Ausbrüchen in Einrichtungen der Kindertagesbetreuung oder Schulen unter Bedingungen der Pandemie und Kozirkulation weiterer Erreger von Atemwegserkrankungen “ [9] als Grundlage der Indikationsstellung herangezogen worden.

Initial war die Abstrichempfehlung seitens des RKI zurückhaltend, von Ka- lenderwoche 10 bis 19 erhielten maximal $33 \%$ (Mittelwert: 8\%) der Konsultationen wegen respiratorischer Infekte eine SARS-CoV-2-Diagnostik. Ab der 20. Kalenderwoche erhielt dann, gemäß geänderter Empfehlung, die Mehrheit der Patienten (Mittelwert: 75\%) den Abstrich für die rtPCR-Diagnostik. Ab der Kalenderwoche 34 änderte sich die Indikationsstellung und wurde deutlich zurückhaltender; nur im Allgemeinzustand beeinträchtigte Patienten wurden abgestrichen, sodass maximal $50 \%$ (Mittelwert: $27 \%$ ) der Konsultationen wegen respiratorischer Infektionen einen $\mathrm{Ab}$ strich erhielten.

\section{Diskussion}

Im Lockdown (KW 12-18) zeigte sich in den Kinder- und Jugendarztpraxen im Saarland ein deutlicher und teils massiver Rückgang der Gesamtfallzahlen, aber 
auch der Fallzahlen der präventiven Medizin (Vorsorge und Impfungen) sowie auch der fachärztlichen Versorgung.

Dieser starke Rückgang der Gesamtfallzahlen im Lockdown ist im Saarland auch für den Rettungsdienst (je nach Rettungsmittel $-16--23 \%$ ) und die zentrale Notaufnahme eines maximalversorgenden Krankenhauses $(-34 \%$, bei den unter 30-Jährigen $-45 \%$ ) dokumentiert [5]. Auch national wurden bereits in mehreren Veröffentlichungen massive Fallzahlrückgänge während des Lockdowns und der COVID-19-Pandemie, v. a. in der Notfallversorgung, gezeigt $[10,14,17]$.

Internationale Veröffentlichungen zeigen während der COVID-19-Pandemie Rückgänge der Impfquoten [4, 12]. Für den Aspekt der Kindervorsorgeuntersuchungen zeigte eine Auswertung des Berliner Kindervorsorgeregisters vergleichbare Effekte wie in der vorliegenden Untersuchung: Einem stabilen Verlauf im Januar-Februar folgte März-Mai ein Rückgang und im Juni ein Aufholeffekt [3]. Beide Bundesländer besitzen ein zentrales Einladungssystem für Kindervorsorgeuntersuchungen.

Was die Früherkennungsfallzahlen betrifft, so ist es mit dem Lockdown zunächst $\mathrm{zu}$ einem starken Einbruch gekommen, der jedoch deutlich geringer ausgeprägt ist als der Rückgang der Gesamtfallzahlen. Ursachen hierfür lagen sowohl bei den Eltern (Absagen von Terminen aus Sorge vor Infektionsrisiken) als auch in der Anpassung des Praxismanagements auf die Pandemie.

Betrachtet man nur die Früherkennungsfälle, folgte einem initialen Rückgang ein fast vollständiges Aufholen bis Ende des ersten Halbjahres. Insgesamt ist somit ein nachhaltiger Kollateralschaden durch nichterfolgte Früherkennungsleistungen sehr unwahrscheinlich.

Im Herbst 2020 ist die Nachfrage nach der Influenzaimpfung, auch nach entsprechenden Empfehlungen durch Politik und Infektiologen in den Medien, sehr hoch. In der Praxis des Erstautors wurde mehr Impfstoff, als für die Saison 2019/2020 benötigt, bezogen, und zum Zeitpunkt der Manuskripterstellung ist dieser bereits vollständig verbraucht bzw. verplant. Die Nachfrage besteht noch fort.
Mit Stichtag zum 15.09.2020 hat der BVKJ Landesverband Saarland im Rahmen der honorarpolitischen Arbeit bei den Praxen nochmals die Differenz der Gesamtfallzahlen sowie Früherkennungsfallzahlen für das 3 . Quartal bis zum Stichtag erfragt. 25 der 52 Praxen (48\%) haben ihre Gesamtfallzahldifferenz gemeldet, deren Median bei $-1,07 \%$ lag, und 21 der 52 Praxen (40\%) die Früherkennungsfalldifferenz, die bei $-1,6 \%$ im Vergleich zum Vorjahr lag.

Die fachärztliche Versorgung betreffend ist es nach dem Lockdown ebenfalls zu gewissen Aufholeffekten gekommen, jedoch war der Rückgang im Lockdown größer als bei den Früherkennungsleistungen, sodass hier kein vollständiges Aufholen bis zum Ende des ersten Halbjahres zu verzeichnen war. Hier besteht grundsätzlich ein erhöhtes Risiko für Kollateralschäden, wenn Kinder und Jugendliche mit schwerwiegenden Grunderkrankungen aufgrund der Pandemie nicht mehr in gleichem Umfang von entsprechenden Schwerpunktpraxen und Spezialambulanzen betreut werden können oder das Betreuungsangebot aus Angst vor Ansteckung nicht wahrnehmen.

In der Praxis des Erstautors war ein abrupter Konsultationsrückgang bei respiratorischen Infektionen während des Lockdowns zu verzeichnen, wie auch in nationalen wie internationalen Arbeiten $[2,6,8,11]$ gezeigt. Das zweite Quartal dauerte von Mitte KW 14 bis KW 27, und dieser Effekt ist vermutlich eine der Ursachen für den Rückgang der Gesamtfallzahlen in den Praxen. Es ist sehr wahrscheinlich, dass durch die Präventionsmaßnahmen im Hinblick auf die Übertragung der SARS-CoV-2-Infektion auch das Risiko einer Übertragung anderer Atemwegsviren sinkt. Trotz des fäkaloralen Übertragungsweges zeigte sich ein vergleichbarer Effekt auch bei Gastroenteritiden. Im Sommer wurden viele Aktivitäten ins Freie verlagert.

Während des Lockdowns und der Schließung von Kinderbetreuungseinrichtungen ist es bei 2 häufigen ambulanten pädiatrischen Infektionen zu einem erheblichen Konsultationsrückgang gekommen.
Vom zeitlichen Verlauf her gewinnt man den Eindruck, dass es mit der Wiederaufnahme eines Regelbetriebes in den Kindergemeinschaftseinrichtungen zu einem Wiederanstieg der respiratorischen Infektionen sowie Gastroenteritiden gekommen ist. Eine Gruppe aus Großbritannien zeigte dies ebenfalls für die Atemwegsinfekte [13].

Der Eindruck in der Praxis, auch im Austausch mit Kollegen, war jedoch, dass hier auch viele Konsultationen aus Verunsicherung der Eltern bzw. auf Druck aus den Gemeinschaftseinrichtungen erfolgt sind, die in anderen Jahren bzw. bei anderen Umständen nicht erfolgt wären (banale Infekte führen zu einer Konsultation). Verstärkt wurde der Eindruck dadurch, dass kaum Kinder schwer erkrankt waren, z. B. stationär eingewiesen werden mussten.

Für einen Nachweis der Kausalität des Anstiegs der Infektionen nach Wiederöffnung der Kinderbetreuungseinrichtungen sind weitere Studien erforderlich. Die multiplen Auswirkungen der Schließungen sind derzeit Gegenstand nationaler wie internationaler Diskussion und Forschung $[1,7,16]$.

\section{Fazit für die Praxis}

- Der Lockdown hat in allen untersuchten Versorgungsbereichen der pädiatrischen Praxen zu deutlichen und teils massiven Fallzahlrückgängen geführt.

- Im Teilbereich der Früherkennungsuntersuchungen (Kindervorsorgeuntersuchungen und/oder Impfungen) sind diese ausgefallenen Konsultationen fast vollständig bis Ende des ersten Halbjahres nachgeholt worden.

- Mit Beginn des Lockdowns ist es zu einem abrupten und anhaltenden Rückgang der Konsultationen wegen respiratorischer und gastrointestinaler Infektionen gekommen. 


\section{Korrespondenzadresse}

Karsten Theiß
Kinder- und Jugendarztpraxis
Theiß
$\begin{aligned} & \text { Oststraße 68, 66386 St. } \\ & \text { Ingbert, Deutschland } \\ & \text { karsten.theiss@kinder-und- } \\ & \text { jugendarzt-theiss.de }\end{aligned}$

Danksagung. Der herzliche Dank gilt den nachfolgenden saarländischen Kinder- und Jugendarztpraxen, die ihre Fallzahlen herausgesucht und zur Auswertung übermittelt haben; ohne die Unterstützung wäre die Arbeit nicht durchführbar gewesen: Gemeinschaftspraxis Boxberg (Neunkirchen), Gemeinschaftspraxis Goethestraße (Neunkirchen), Gemeinschaftspraxis Talstraße (Homburg/Saar), MVZ Saar Marienhaus - Sophienstiftung GmbH (Saarlouis), Albrecht (Heusweiler), Aliani (Saarlouis), Alt (Saarbrücken), Andler (Eppelborn), Benn (Saarbrücken), Berndt (Saarbrücken), Bitsch (Merzig), Breitenmoser (Saarlouis), Brixius (Homburg), Cartarius/lgel/Gunther (Saarbrücken), Danner-Baltes (Saarbrücken), Ehlayil (Bexbach), Evers \& Saupe (St. Wendel), Holbeck (Merzig), Jost (St. Ingbert), Klein (Saarbrücken), Krämer (Illingen), Lagemann (Merzig), Magar (Saarbrücken), Meier (Saarbrücken), Messerle \& Kreckmann (Riegelsberg), Neumeier-Schmitt (Kleinblittersdorf), Niethammer (Völklingen), Rudolph (Homburg), Rüenauver (Sulzbach), Salzmann/ Bützler/Naumann (St. Wendel), Scheffler (St. Wendel), Schwarz (Dillingen), Spaniol (Saarwellingen), B. Ulrich (Saarbrücken) und Weber (Ottweiler)

\section{Einhaltung ethischer Richtlinien}

Interessenkonflikt. K. Theiß, A. Simon, N. Graf und T. Rohrer geben an, dass kein Interessenkonflikt besteht.

Für diesen Beitrag wurden von den Autoren keine Studien an Menschen oder Tieren durchgeführt. Alle beschriebenen Untersuchungen wurden mit Zustimmung der Ethikkommission der Ärztekammer des Saarlandes durchgeführt (Kenn-Nr. 188/20).

\section{Literatur}

1. Angoulvant F, Ouldali N, Yang DD et al (2021) COVID-19 pandemic: Impact caused by school closure and national lockdown on pediatric visits and admissions for viral and non-viral infections, a time series analysis. Clinical Infectious Diseases 72:319-322. https://doi.org/10.1093/cid/ciaa710

2. Baker RE, Park SW, Yang W et al (2020) The impact of COVID-19 non-pharmaceutical interventions on the future dynamics of endemic infections. medRxiv:2020.2006.2022.20137588. https://doi. org/10.1101/2020.06.22.20137588

3. Blankenstein O, Rintisch W, Buchholz T et al (2020) Auswirkungen der SARS-CoV-2 Pandemie auf die Früherkennungs-Untersuchungen bei Kindern. Klin Padiatr. https://doi.org/10.1055/a-1247-5119 (Online ahead of print)

4. Bramer CA, Kimmins LM, Swanson R et al. (2020) Decline in Child Vaccination Coverage During the
COVID-19Pandemic-Michigan Care Improvement Registry, May 2016-May 2020. MMWRMorb Mortal Wkly Rep 69:630-631. https://doi.org/10.15585/ mmwr.mm6920e1

5. Braun C, Schlechtriemen T (2020) Auswirkungen des Corona-Lockdowns auf die prä- und innerklinische Notfallversorgung. Saarländisches Ärztebl 73(9):8-16

6. Buchholz U, Buda S, Prahm K (2020) Abrupter Rückgang der Raten an Atemwegserkrankungen in der deutschen Bevölkerung. Epidemiol Bull 2020(16):7-9. https://doi.org/10.25646/6636.2

7. Christakis DA, Van Cleve W, Zimmerman FJ (2020) Estimation of US children's educational attainment and years of life lost associated with primary school closures during the Coronavirus disease 2019 pandemic. JAMA Netw Open 3(11):e2028786. https://doi.org/10.1001/jamanetworkopen.2020. 28786

8. Cowling BJ, Ali ST, Ng TWY et al (2020) Impact assessment of non-pharmaceutical interventions against coronavirus disease 2019 and influenza in Hong Kong: an observational study. Lancet Public Health 5(5):e279-e288. https://doi.org/10.1016/ S2468-2667(20)30090-6

9. Deutsche Gesellschaft Für Pädiatrische Infektiologie (Dgpi), Deutsche Gesellschaft Für Kinder- Und Jugendmedizin (Dgkj), Deutsche Gesellschaft Für Krankenhaushygiene (Dgkh) et al (2020) Maßnahmen zur Aufrechterhaltung eines Regelbetriebs und zur Prävention von SARS-CoV-2-Ausbrüchen in Einrichtungen der Kindertagesbetreuung ode Schulen unter Bedingungen der Pandemie und Kozirkulation weiterer Erreger von Atemwegserkrankungen. https://www.dakj.de/wp-content/ uploads/2020/08/DAKJ-SN-AufrechterhaltungRegelbetrieb-Gemeinschaftseinrichtungen.pdf. Zugegriffen: 17. Aug. 2020

10. Greiner F, Kocher T, Schirrmeister W et al (2020) Inanspruchnahme deutscher Notaufnahmen während der COVID-19-Pandemie - der Notaufnahme-Situationsreport (SitRep). Epidemiol Bull 2020(27):3-5. https://doi.org/10.25646/6959

11. Luo Z, Li S, Li N et al (2020) Assessment of pediatric outpatient visits for notifiable infectious diseases in a University Hospital in Beijing during COVID-19. JAMA Netw Open 3(8):e2019224. https://doi.org/ 10.1001/jamanetworkopen.2020.19224

12. McDonald HI, Tessier E, White JM et al (2020) Early impact of the coronavirus disease (COVID19) pandemic and physical distancing measures on routine childhood vaccinations in England, January to April 2020. Euro Surveill 25(19):2000848. https://doi.org/10.2807/1560-7917.ES.2020.25. 19.2000848

13. Poole S, Brendish NJ, Tanner AR et al (2020) Physical distancing in schools for SARS-CoV-2 and the resurgence of rhinovirus. Lancet Respir Med 8(12):e92-e93. https://doi.org/10.1016/S22132600(20)30502-6

14. Ramshorn-Zimmer A, Schröder R, Fakler J et al (2020) Notaufnahme während der Coronapandemie: Weniger Non-COVID-19-Notfälle. Dtsch Arztebl 117(24):1201-1205.

15. Robert Koch-Institut (2020) COVID-19-Verdacht: Maßnahmen und Testkriterien - Orientierungshilfe für Ärzte. https://www.rki.de/DE/Content/ InfAZ/N/Neuartiges_Coronavirus/Massnahmen_ Verdachtsfall_Infografik_Tab.html. Zugegriffen: 23. Okt. 2020

16. Schlack R, Neuperdt L, Hölling $H$ et al (2020) Auswirkungen der COVID-19-Pandemie und der Eindämmungsmaßnahmen auf die psychische
Gesundheit von Kindern und Jugendlichen. JHealth Monit 5(4):23-34. DOI: 10.25646/7173

17. Tschaikowsky T, Becker Von Rose A, Consalvo Set al (2020) Patientenzahlen im Rahmen der COVID-19 Pandemie in einer zentralen Notaufnahme. Notfall Rett Med. https://doi.org/10.1007/s10049-020 00757-w (Online ahead of print) 